Conceptualising Health Information Seeking Behaviours and Exploratory Search: Result of A Qualitative Study

Patrick Cheong-Iao Pang

Department of Computing and Information Systems, The University of Melbourne

NICTA Victoria

cipang@unimelb.edu.au

Karin Verspoor

Health and Biomedical Informatics Centre, The University of Melbourne

Department of Computing and Information Systems, The University of Melbourne

karin.verspoor@unimelb.edu.au

Shanton Chang

Department of Computing and Information Systems, The University of Melbourne shanton.chang@unimelb.edu.au

Jon Pearce

Department of Computing and Information Systems, The University of Melbourne j.pearce@unimelb.edu.au

Abstract

The Internet has become an important source of health information for many people, including nonmedical experts. While much health information is provided through the Internet, such information is often spread across multiple websites and providers. With the decentralised nature of the Internet, people are often unable to access health information at a single point, and thus extra efforts are needed for seeking information with search engines. The information seeking process is a crucial step for obtaining the needed health information. According to prior research, seekers apply an exploratory approach in the information seeking process of health-related topics. Although information seeking behaviours have been studied extensively in past research, few details specifically about information seeking in the health context can be found. This paper reports on a qualitative study that investigates the information needs and behaviours of health information seekers. Based on the results of the study, we present a model conceptualising health information seeking behaviours as well as the characteristics of the exploratory search approach observed in the health information seeking process.

Keywords: search approach, exploratory search, consumer health information, information seeking behaviour

\author{
The final publication is available at Springer via \\ http://dx.doi.org/10.1007/s12553-015-0096-0
}




\section{Conceptualising Health Information Seeking Behaviours and Exploratory Search: Result of A Qualitative Study}

\section{Introduction}

The Internet is a source of a range of different health information resources for non-medical experts, from official health websites, private health service providers, personal blogs, to discussion forums. Past studies have demonstrated that the use of online resources for obtaining health information is increasing [1, 2]. As the demand for online health information grows, it is crucial to understand the motivations underlying these information seeking behaviours, the information needs of the seekers, the strategies for seeking information chosen by the seekers.

The World Wide Web is designed as a decentralised network. Health information, even on the same general topic, is available from many different websites and providers. For example, descriptions about Hepatitis B can be obtained from government websites, the website of medical centres, or even Wikipedia. Without a single source of information, this decentralised nature makes search engines the primary mechanism for accessing online information - i.e., people choose to search for the information in the first instance. Shown in a study, $77 \%$ of health information seekers in fact use search engines [2].

Getting health information from the Internet is like finding a needle in the haystack. Especially when dealing with unfamiliar and unknown problems [3], as is often the case for health-related concerns [4], the search approach employed becomes more exploratory [5]. In contrast to simply submitting a search query, exploratory search involves a series of cognitive learning and query reformulation processes. With exploratory search, a more complete picture of the knowledge domain is being built during the search, which implies the existence of both learning and investigating activities. Although exploratory search can be found in different search tasks, there is only limited understanding of exploratory search in the health context.

In order to have a more complete understanding about online information seeking and search approaches taken in the consumer health context, we conducted a qualitative study to observe participants' behaviours. We discover that in many cases people take an exploratory approach to find relevant information. In addition, based on the activity logs recorded in the study, we summarise the patterns of participant behaviours into a model with four categories. Information seekers in these categories have different levels of research tactics and reading engagement. The model aims to provide a perspective from the angle of behavioural outcomes, so that health website owners and information retrieval (IR) specialists can improve their products in terms of user needs. Some design implications are identified as well at the end of this paper.

This paper is structured as follows: it firstly summarises the current research and trends in obtaining online health information, followed by a report on the result of our qualitative study. Drawn from the results of the study, we propose a model for conceptualising online health information seeking behaviours, as well as the characteristics of the exploratory search approach observed in the health information seeking process.

\section{Literature Review}

\subsection{Online Health Information Seeking}

More and more people choose to obtain health information from Internet websites. According to a 2013 study, $72 \%$ of U.S. Internet users have tried to access online health information, and $77 \%$ of these people started with a search engine [2]. A study on search engine logs also showed that $7.5 \%$ of submitted queries were related to 
medical or health information [6]. Research discovered that majority of Internet users acquire health information via search engines $[2,7]$.

Past studies have found that Internet users look for a wide range of health information [1, 2, 8-13], including information about specific medical conditions or symptoms, causes and treatments of diseases, diet information and healthy lifestyle tips, etc. People are also seen to look for support and extra information from the Internet at different stages of a serious health problem [14, 15]. The diverse types of health information demanded reflect differences in information needs, resulting in different seeking behaviours.

\subsection{Health Information Needs}

Information needs arise when people realise their existing knowledge is inadequate to satisfy their goal [16], or feel stressed while facing threats such as health problems [17, 18]. Gorman [19, 20] classified information needs into four categories: recognised, pursued, satisfied and unrecognised, showing how people perceive and engage with the needs. For health information seeking tasks, Alzougool et al. [21, 22] classified health information needs into combinations of recognised/unrecognised and demanded/undemanded. The recognised/unrecognised factor reflects if an individual clearly realises the motivation of getting certain information, or just causally receives the message. The demanded/undemanded factor specifies whether an actual information seeking behaviour is carried out for the corresponding needs.

\subsection{Information Seeking Behaviours}

Scholars suggest information seeking behaviours are stimulated by information needs. Wilson $[17,18]$ argues that people seek additional information to relieve stress and uncertainty when they face critical situations. The information seeking process is also known as a sense-making process [23]. Case [16] illustrates this process as bridging a knowledge gap: the missing and needed information is analogous to a valley in the middle of knowledge mountains. The seeker looks for extra information to "build" a bridge across the gap [16].

There are many theories to describe the process of information seeking. Wilson has identified four modes of information seeking behaviours. In addition to searching, people also acquire information - meaning they passively receive information even if they do not realise specific information needs [17]. Kuhlthau suggests an information search process (ISP) model with six stages to describe the behaviour of seekers [24]. The model emphasises changes of seeker thoughts and feelings in the information seeking process. Ellis et al. describe the process of satisfying information needs as a linear sequence of behaviour [25-27]. Based on these works, Lambert and Loiselle [28] summarise literature about health information seeking behaviour and identify various connections between information needs and information seeking behaviours.

\subsection{Exploratory Search}

Recently exploratory search has gained a lot of focus among researchers about search and information seeking behaviour. Marchionini [5] defines exploratory search as a search approach that incorporates learning and investigation along with the actual lookup activity. Exploratory seekers are often unfamiliar with the knowledge domain of their search targets [29], unsure about the ways to achieve their goals [29], or need to address unfamiliar or unknown problems [3]. As health information seekers have only limited knowledge in the medical context [30], exploratory search is one of the characteristics observed in the health information seeking process [4]. Cartright et al. [31] suggest exploratory health search can be further broken down to evidence-based and hypothesis-directed. Also, Zarro [32] argues health-related exploratory search can be analysed from a social psychology perspective. Although exploratory search has been investigated from different aspects, as we can see, there is few research combining health topics with exploratory search to draw a comprehensive understanding in the consumer health context. 


\section{The Qualitative Study}

This research aims to capture an in-depth understanding of online health information seeking behaviours, as well as the underlying information needs that motivate the behaviour. To facilitate data collection, we conducted a qualitative study to observe the actual behaviours of health information seekers. This section introduces the design of this study and the results we have concluded.

\subsection{Research Design}

We chose a qualitative approach in this research. As previous studies cannot provide a detailed view on individual information seeking behaviours, a qualitative study allows us to collect a rich dataset and to make new insights [33].

The main component of the qualitative study is a lab observation. Lab observation has been used in a range of research to investigate human behaviours in regard to health issues [7, 34-36]. While some research uses a think-aloud approach to collect participants' thinking during an experiment, we used a debrief session instead to avoid disruption and slow down of the entire flow of the experiment [33].

The entire lab session was structured as follows: the first section was a semi-structured interview about their past experiences of finding health information on the Internet; in the second section participants were given access to a computer to find online health information for two pre-defined tasks, with screen activities and web browsing histories recorded; the last section was a debriefing interview which is a semi-structured interview for understanding how participants performed the tasks in detail. Each lab session lasted about an hour.

The search tasks represented two styles of common health scenarios. This setup was inspired by the causes and understanding of exploratory search. We hoped to show the behavioural differences between general and exploratory search with a contrastive setup of tasks by leveraging the clarity and abstraction of the tasks. In one scenario, participants were asked to find information on how to care for a diabetic family member. This simulated an information need following a straightforward diagnosis from a medical professional. In another case, participants were tasked with identifying information to append to a Wikipedia page on urination problems and their symptoms. Participants needed to find out the relevant information from other sources in the web with limited and vague symptoms provided. The first scenario was designed with the aim of observing seeking behaviours for explicit recognised needs, whereas the second targeted for unrecognised needs with a more vague description of a health problem. To avoid the bias of using a search engine at the first scene, we cleared the home page and all browsing history in the browser prior to each session to ensure the current participant's behaviour was not affected.

Feedback from interviews and web browsing activities were analysed accordingly. Interviews were transcribed in full and reduced to a number of codes iteratively [37]. Data relevant to search approaches, search motivations and information seeking behaviours were organised into themes. Themes were then derived with the thematic analysis methodology [38]. The methodology involved reading transcripts multiple times, categorising codes into themes and refining the theme list iteratively along the analysis. The number of participants connected to each theme was tracked for verifying the generality of themes.

A web browser plugin was developed to record web browsing activities during the lab session. The plugin produced a plain text $\log$ with the web addresses of visited pages, time of visits, duration of each visit, and identifiers of sessions and tabbed browser windows. For each participant, a navigation graph was created manually with this information. A navigation graph indicates the flow of web page views along the search progress, as well as the duration of each step. This can provide us insights about how the search process and the 
behaviours evolve while the participants engage in the seeking process. Navigation graphs with similar patterns were merged into themes to for further derive the characteristics of the behaviour.

\subsection{Overview of Participation}

The lab session was set up in the period of October to December of 2013. We used multiple methods to recruit participants. Posters were presented at locations across the university campus. E-mail invitations were sent out to staff and students to encourage participation. Participants were also allowed to invite potential participants to our study through their connections. Recruitment continued until data saturation was achieved. This experiment was voluntary and no incentive was given to participants for the study.

In total 20 participants completed our lab experiment (11 male; 9 female). In terms of identity, they comprised 8 students, 9 university staff and 3 external participants. All reported that they were capable of reading and searching information on the Internet. All participants claimed they had experience in searching health information online. For privacy issues, age group was recorded instead of exact age of participants. The distribution of age groups is listed in Table 1.

Table 1. Age group distribution of participants

\begin{tabular}{|l|l|l|}
\hline Age Group & N & Percentage \\
\hline $21-30$ & 8 & $40 \%$ \\
\hline $31-40$ & 6 & $30 \%$ \\
\hline $41-50$ & 4 & $20 \%$ \\
\hline Over 50 & 2 & $10 \%$ \\
\hline
\end{tabular}

\subsection{Results}

This section reports on the data gathered in the qualitative study. Firstly we present the observation of the lab experiment and list the themes that emerged after the analysis of interview transcripts. Then, we present the behavioural patterns of our participants. These patterns were observed from the navigation graphs produced from the activity logs.

During the review of the data collected in our study, we segmented the dataset into age groups and by gender. The analysis of the partitioned data shows no significant themes and patterns across different groups of participants. Therefore, the results and discussions in this paper apply to general health information seekers, regardless of their age and gender.

\subsubsection{Current Health Information Seeking}

We first reviewed participants' feedback on how to facilitate their health information seeking. We observed that 19 out of 20 participants used Google to look for health information. Google was used in two ways. In addition to its search function, Google was utilised as a web directory by our participants. In some sessions participants clearly knew the name but not the address of the website they wished to access, and they used Google to directly jump to the website. These are known as navigational queries in the literature [39]. In addition, Google was identified to act as a "glue" connecting different parts of a single website. There were occasions that the participants could not find, or simply missed, a hyperlink. Then they returned to Google to search for the information. An example illustrating this could be accessing another article within Wikipedia when the targeted article was not linked in the current document. 
Our observation suggested that search engines may not the best tool for health searches. Knowing accurate keywords for medical issues was a problem. As one of the participants said: "I do not know sometimes what are the right keyword to use in order to get the right information." (Participant \#1) Also, participants mentioned the difficulty of filtering and narrowing down the search results because of the lack of medical knowledge and the analytical skills: "If you search for health, then there is so much information coming up. You are not the doctor, so you pick and choose whatever you want and that could be very wrong at the end, because you think you're sicker. It all depends on what kind of symptoms you searched for." (Participant \#13)

Participants seemed to use multiple websites for health topics in their search sessions. The reason was mainly about the trustworthiness of the online health information and a reassuring purpose. "Sometimes you don't know whether it's genuine so I keep searching other sites for that." (Participant \#1)

\subsubsection{Search vs. Explore}

Seekers demonstrate different approaches to look for health information with different information needs, and sometimes they demonstrate a more exploratory approach in the search process. As reported by participants, generic search is a more focused one with a clearer target of what is being looked for in mind. In contrast, health search can lead to an exploratory behaviour where the search target is more fuzzy, and the seeker is more eager to read new information on the Internet.

"Searching tends to be focused. I feel like when I am searching, I have my keywords in my head, I have a reasonable idea of what I am looking for or find. So a lot of time I already go with some sort of information in keywords... Exploration is not so focused. Exploration suggests that you have more time. You are not going in so many keywords perhaps. You are much open to the information that's coming to you." (Participant \#6)

The exploratory approach of search is not just finding the needed information but also using the visible connections (e.g. hyperlinks or recommendations of further readings) in the current website to learn more.

"For me searching is like Google and find the website; and exploring is taking time to read the information and check the information a website has, and learning. My perception of exploring would be more on the learning experience. Searching is just getting where to find the information... Because as I Google, I can find other words and connections that I can use to search." (Participant \#7)

Although we distinguish the behaviour of focused and exploratory search approaches, some participants suggested they were not completely distinct. They reported the two types of search co-existed and alternated at the same time.

"At the beginning I was searching, and then I started exploring things more and more, and opened various links. I think when I open multiple links that's kind of exploring. Then I did another search, which is more specific and then I continue exploring. " (Participant \#17)

\subsubsection{Why do Seekers Explore?}

Most people seek online health information because of health problems. As reported by most of the participants, encountering health issues was one of the factors that drives them towards the exploratory approach. Note that the participants did not simply perform a simple search, but experimented with different queries for exploring more around the issue, for the purpose of thoroughly understanding the health issue.

"I would usually search if I think there is a health problem... If I go search for something, I don't stop at the first page comes up at Google. I explore, I read further, try different search terms, looking at the other results... 
And then occasionally I will think of new words, I go back to the search bar and type that different words." (Participant \#2)

Participants were observed to be more exploratory if they encountered conflicting information. This is very common in the world of the Internet because everybody can post online without necessary verification on the information. For resolving the conflict in seekers' minds, they investigated different data sources, followed hyperlinks to different articles, read carefully the contents, and tried to verify the reliability of the information.

"[When] I receive advice that seemed to be conflicting from different sources I often look for an answer... I cross [check] references, check another source... One thing leads to other things. They have links within the article, and I end up finding about more information than needed, still interesting and related." (Participant \#20)

Discovered in the lab observation, curiosity made participants more exploratory in their efforts to find health information on the Internet. While curiosity is often triggered passively, e.g. hearing a celebrity being diagnosed with certain health problem, it appears that the exploratory health information search may last for a longer period of time as there is no urgent need to retrieve the information immediately. One may resume the seeking task later on whenever the time is allowed.

"Usually I end up finding information on the government websites and specific to the health information I am curious about... It lasts probably a few days. Because sometimes I started with one bit of information and I keep searching other websites as well and looking deeper to the topic. Just to get more information and a bigger picture - to see if there [are] any other related issues." (Participant \#4)

Finally, participants seemed to display an exploratory approach when the health issue was related to people that were important to them, such as family members. They felt stronger urgency and determination to read as much information as possible in this scenario.

"But I guess if it was my mum like that, I would go back to look into, to compare information on sites... I would definitely look for more websites... I think I will continue to search afterwards as well." (Participant \#13)

\subsubsection{Behavioural Patterns}

During the experiment, participants were required to complete two tasks of finding health information. A navigation graph was generated from web browser logs for every successful task. In total 36 graphs were created while 4 tasks not completed by participants due to time constraints on these lab sessions. These graphs were further analysed and categorised according to the activity patterns. These patterns are reflecting the information seeking behaviours and choices of web navigation in the seeking process. We have identified four categories of behavioural patterns by reviewing all navigation graphs (Figure 1). 
Type A:

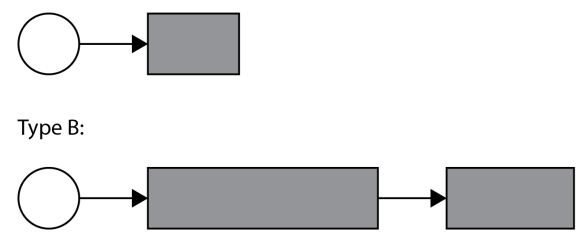

Type C:
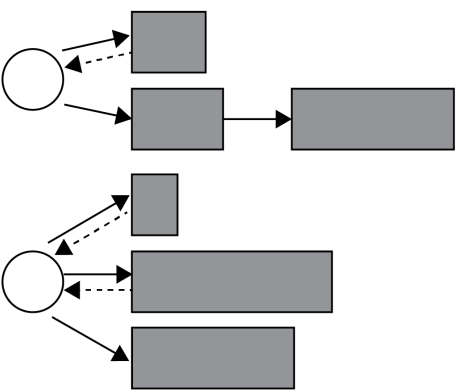

$\bigcirc$ Search Query
Type D:
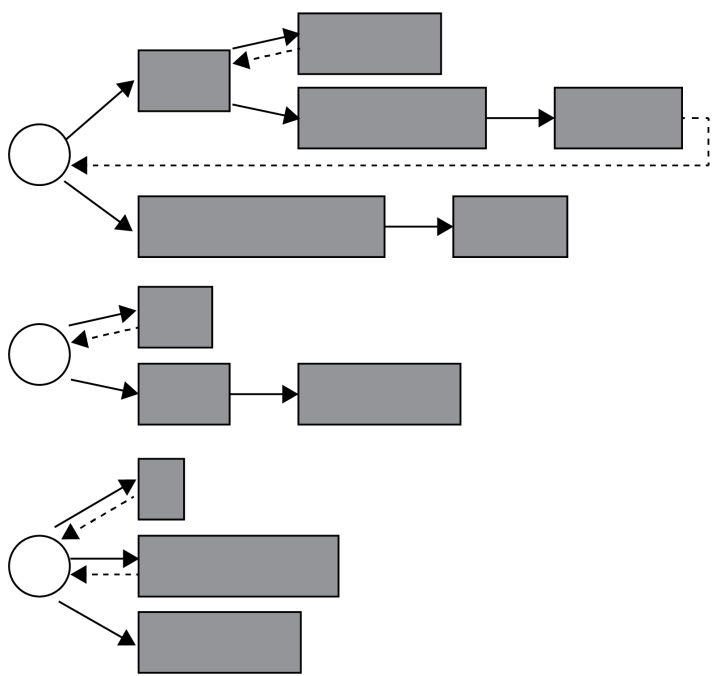

$\longrightarrow$ Hyperlink Follows

4-- Back to Previous Page

Web Page Reading (Bar Width Denotes Reading Duration)

Figure 1. Health information seeking behaviours

Type A shows the most simple pattern of all health information seeking behaviours. The seeker simply sends a keyword query to a search engine. In the list of related websites that returned from the search engine, he/she skims the one which is believed to be most relevant. The seeking process stops quickly once the information is found. It is also noticeable that the seeker does not engage in deeply reading the web page.

Type B looks very similar to Type A in terms of the small number of searches incurred in the information seeking process. However, the seeker engages in longer and deeper reading activities. Identically to Type A seekers, they pick the most suitable web page to start with; then they behave differently in follow-up of hyperlinks and the readings of subsequent web pages. The duration of reading is longer as the seeker digests and absorbs the information. The series of reading continues until no more valuable information is found.

Seekers of Type $\mathrm{C}$ demonstrate not much reading but more query reformulations. In such a scenario, the seeker wishes to explore additional information. Most of the time, he/she tries to skim through the search results and picks up only web pages that are relevant to the information need. While the search goes on, the seeker will adjust the query keywords with the information read and perform a new query, if the overall search result is not satisfactory. Sometimes the seeker may follow hyperlinks to seek for more information. However, the steps of hyperlink follow-up are limited since new search queries are more preferred for this category of seekers.

Type D seekers use a mixed approach to find health information. This type of seekers begins with a keyword search and chooses a small number of good websites (usually contain large amount of content and reputable) for examination. If needed, they use hyperlinks to discover new information page-by-page. They are observed immersing themselves into the reading of each page. Moreover, they also make use of search engines to explore additional information. A reformed query is executed and the seeker goes over the search result again. In short, this type of seekers displays a multiple iterations of both searching and reading until the information need is satisfied.

To sum up, these four categories of patterns show us the different behaviours of health information seekers. We observe that some participants did not read in-depth, whereas the others studied the topic extensively. These 
variations can provide hints for distinguishing different types of health information seekers, which we discuss in the next section.

\section{Discussion}

Based on the results of our qualitative study, we further discuss the phenomenon of exploratory search approaches taken during health information seeking processes. We propose a model to conceptualise the health information seeking behaviours and derive design implications for health websites to provide better support for seekers' needs.

\subsection{Problems with Current Health Information Seeking}

Everyday use of the Internet heavily relies on search engines. Search engine giants such as Google have become part of people's lives. Most of modern search engines are designed to work with keyword queries and have no special adaptation for health-related searches. According to studies, lay-people usually have only limited knowledge of the medical domain [30], and face difficulties in searching due to insufficient knowledge of technical and medical language [40,41]. We have identified similar problems in this study. Participants did not know the keyword for searching for some tasks, due to the lack of medical terminologies (such as not knowing the name of a disease or misspelling certain medical terms) or simply not knowing what keywords to start with. Moreover, they were not able to query precisely and narrow down the search results. For example, for a given set of symptoms, search engines returned multiple possibilities and the participants could not determine the precise one that they wished to read.

Irrespective of the scenario, almost every participant searched with Google. They explicitly entered the Google web address or used the search toolbar even when we removed the default home page setting. This reflects the high tendency of using search engines in health information seeking. Some participants reported that they used to save bookmarks of health websites to retrieve health-related information, but these bookmarks were no longer used after the growth of Google. This behaviour adds findings to the work of Choo et al. [42] that seekers prefer to start their information seeking behaviour (i.e. the "starting" stage) with a search engine nowadays. However, search engines may become a barrier for obtaining the most suitable health information since these search engine algorithms controls the ranking of health websites. Without special handling of health information needs, the algorithms may not know the best way to sort the result list. As a result, seekers may not be able to access a good health website if it is displayed in a lower rank; or search engines may even hide a website, though it contains pieces of creditable and useful information.

There were some cases in which the participants clearly knew which website to go to but did not know its web address. Google was used to jump to a particular website, described as a teleport process in the work of Teevan et al. [43]. Also, Google was linking different web pages so that the user could navigate from one to another, which was acting as glue of multiple web pages within a website. Some participants felt frustrated in navigating in a website because of an unsatisfactory design (such as unobvious or hidden hyperlinks or menu items), therefore they used Google to locate the information. This implies the necessity of good and intuitive design when setting up a health website.

\subsection{Factors Contributing Exploratory Search}

Health information seeking behaviour, or health search, is different from search of other topics in many ways. It occurs for dealing with health-threatening situations, making medical decisions, and behavioural changes and preventative measures [28]. Based on our observations, this section illustrates a number of factors that lead to an exploratory search approach when people look for health information. 
Participants with health problems or being diagnosed with a certain sickness were found to be more exploratory in the search process. They had explicit information needs on certain aspects, such as treatments and remedies. In this case, they took an exploratory approach of search for understanding the complete picture of the situation or getting more options for facing the health problem. Therefore, the exploratory approach serves as an outcome to the stress/coping theory $[17,18]$ as the seeker finds information in an exploratory way to cope with the stressful situation. This also denotes that people with recognised needs $[21,22]$ of a particular health condition will lead to relatively exploratory seeking behaviour.

The exploratory approach is found in search when the health issues under investigation are related to people of importance to the seeker. For example, an individual finds and explores as much information as possible if his/her children are sick. This phenomenon is closely connected to the emotional investment. People tend to dive deeper into a problem if it is more directly relevant to them. The level of concern is high at this moment. They would use all potential sources to understand the problem, look for remedies and learn to support ill person. Finding information for important ones is also an unrecognised health information need [21, 22], which is identified to trigger exploratory search [4].

Curiosity is another type of information need that contributes to the exploratory health search. Such information seeking behaviour is usually triggered in passive scenarios, like hearing of pieces of information about health when watching TV, or becoming interested in a medical topic through a conversation. Although in this scenario the seeker may have a clearer search target, the search is easily changed into exploratory as he/she may encounter other interesting material throughout the process. This also corresponds to the alternating modes of focused and exploratory search which are introduced in the next section.

\subsection{Exploratory Information Seeking Behaviours}

Based on the result of interview themes, we conclude that exploratory information seeking behaviours in the health context has unique characteristics. Firstly, the search target of exploratory search is not specific. As the interview quotes suggest, focused seekers have a clearer mind and a reasonable plan to search, whereas exploratory seekers need more time to filter out what is needed, narrow down the target and locate the information. This finding is in agreement with the definition of exploratory search established in [5, 29] people need to look up in a wider range of information to satisfy unclear and unfamiliar information needs.

With regards to the range of search targets, participants were seen to use multiple sources to obtain health information. The reasons behind this varied: for confirming the validity of the information, or forming an allaround understanding of the topic. For extending the reading from the current web page and exploring additional information, hyperlinks played an important role as hyperlinks were reported to be more convenient for participants to use. On the other hand, making a new search for extra information requires a cognitive process to form a new query and analyse the result. Seekers are more likely to visit relevant web pages within a site or even external websites by following hyperlinks.

Another characteristic is the desire to understand the information, beyond simply locating the information. The exploratory approach is about discovering and learning new information [5]. We noticed a similar result in our experiment. Participants opened their minds to a wide range of relevant information, and preferred to spend time reading the content to determine the usefulness. Participants reported learning new information encountered during the search that might have been unexpected beforehand. In terms of information needs, a knowledge gap appears at the beginning of the information seeking process [16]. Health exploratory search is the actual behavioural outcome to gradually gather knowledge to "bridge" the knowledge gap.

White and Roth [29] suggest that generic exploratory search will change towards focused search as the search progresses because the uncertainty reduces when the seeker retrieves more information to fill the knowledge 
gap. Using an example of buying a second-hand car: the seeker has no idea at the beginning so he/she finds all car models which fits in the price range - an instance of exploratory search. Then, after examining the result, the seeker looks for details of a particular model. As we can see, the range of the search becomes narrower and focused as the search continues.

We have observed a slightly different behaviour for health information seeking. In the health context, while the information is being learnt, new and unknown information appears at any point. Consequently the level of uncertainty in the seeker's mind will climb again and thus the search will become more exploratory. In contrast to White and Roth's idea of a single direction flow from exploratory to focused search, we argue that these two modes are alternating along the process until the search stops, and the seeker is seen to be interleaving between these two modes.

Figure 2 depicts the alternation between exploratory and focused search. An individual starts the information seeking process with Topic 1 . While he/she may not have a full understanding on Topic 1, exploratory search is adapted and the search area is wider at this time. As the search goes on, the seeker gains more knowledge about the topic. The search target becomes clearer and the search area becomes narrower and focused as the spiral curves indicate in the figure. At any point the seeker may encounter new doubts, this leads a search on a new topic (Topic 2). As the topic is brand-new for the seeker, the search turns into exploratory again. This interleaving process continues until the search stops.

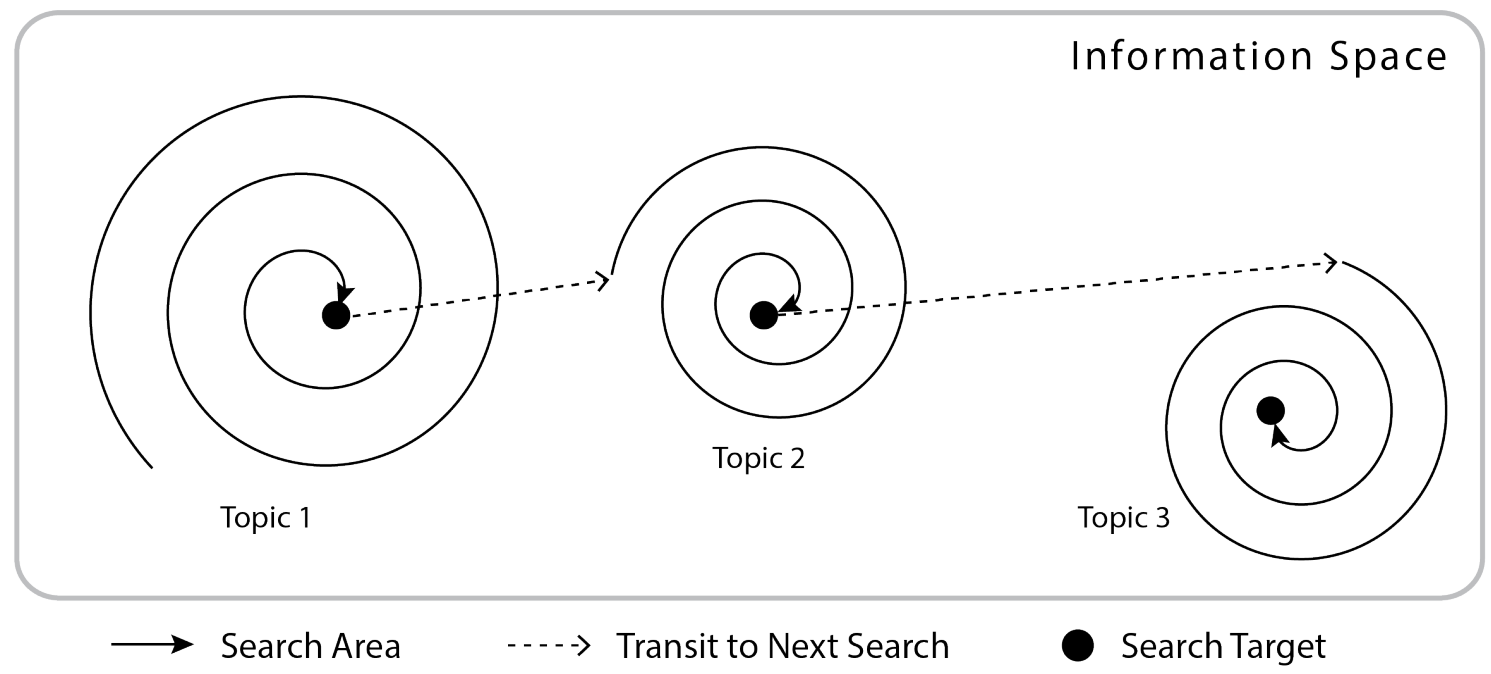

Figure 2. Illustrating alternation between exploratory and focused search

An example from our study can better explain the alternation of search approaches: a participant started with a search for diabetes management information. The search was exploratory at the beginning because he did not know much about this topic. After he learnt that he could control diabetes with eating, he performed a focused search on this area and continued to read. Gradually his uncertainly rose again because he felt confused what kinds of food and cooking methods could be helpful to the disease. At this point his search started to turn back to exploratory and started investigating across a range of topics such as recipes for diabetics, diet suggestions, nutrition information, healthy vegetables, etc.

\subsection{Model of Health Information Seeking Behaviours}

We have introduced the problems and the exploratory nature of health information seeking behaviours in the previous section. The next step is to concentrate these findings into a robust model to describe such behaviours. Taking on an angle from the perspective of designing health websites and enhancing user experience, we put our 
focuses on the seekers' needs of investigating the concerned topic, as well as the reading effort demonstrated in the seeking behaviour.

Figure 3 illustrates our model for conceptualising the online health information seeking behaviour. Drawn from the behavioural patterns observed in our qualitative study, the model presents a combination of seekers' research tactics and levels of reading engagement in a two-by-two matrix. Research tactics represent the eagerness and motivation for digging out in-depth information. For example, a seeker who chases hyperlinks and submits many search engine queries in order to retrieve more information is demonstrating a extensive research tactic. On the other hand, reading engagement measures the duration of reading and the intention of absorbing the information. Skimming and reading just the page summary fall into the group of low engagement while pursuing and digesting the information is considered as high engagement.

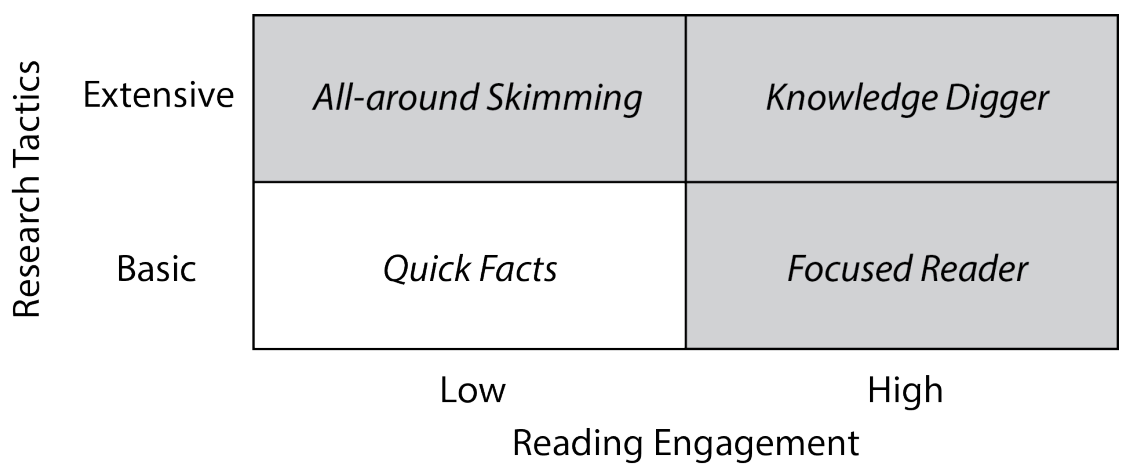

Figure 3. Model of health information seeking behaviours (exploratory approaches are shaded)

Each quadrant in the matrix represents a type of online health information seeking behaviour. Quick Facts seekers (Research Tactics: Basic; Reading Engagement: Low; Lower-left in the Figure) usually have a clearer understand of the topic and know what is missing and needed to learn. They have a concrete information need and go through the material quickly to locate only the part of knowledge that is missing. This is comparable to factual searching in [44] and known item search in [45].

All-around Skimming (Research Tactics: Extensive; Reading Engagement: Low; Upper-left in the Figure) is similar to searches of Quick Facts in the way of skimming articles. Usually this type of seekers has a fair understanding of the problem and wants to investigate an issue more deeply. With the variety of content and quality of online information, the information may not fit the seeker's information at the first scene. They perform a quick read before investing time and effort. In our lab observation, participants with research or professional background took this approach to find health information.

Focused Reader (Research Tactics: Basic; Reading Engagement: High; Lower-right in the Figure) puts a significant effort to read a small number of materials. There are a few scenarios leading to this outcome: lack of skills of proper usage of a search engine; lack of skills to discover external readings within the current web page; or found a lengthy and rich document which meets the information need (such as a journal paper or a PDF leaflet). Besides that, this category of seekers aims to improve their intellectual understandings [44] rather than retrieve facts.

Finally seekers in the Knowledge Digger quadrant (Research Tactics: Extensive; Reading Engagement: High; Upper-right in the Figure) are willing to use a lot of time and effort to investigate the issue they are facing. At the same time, they demonstrate relatively better skills at discovering and exploring for more possibilities to enhance the understanding of the issue. In general, both the reading duration and the number of search queries are high for these seekers. 
Though four types of information seeking behaviours show different patterns on the actual investigation and reading activities, three of them demonstrate an exploratory nature in the search process (shown as shaded in the figure). Exploratory search emphasises the element of learning new knowledge and investigating the knowledge domain [5]. The behaviours of seekers in the highlighted quadrants present one or both these elements. With this point aligning to prior research $[4,31,32]$, we argue that health information seeking, except for those with purposes of retrieving quick facts, is presenting an exploratory search in nature. For health websites, in addition to design for better research activities and reading efforts, it is parallel to consider supporting information exploration and discovery for health seekers.

\subsection{Design Implications}

Drawn on the abovementioned findings, we propose two areas of improvements for enhancing the user experience of health information seekers. These are (1) assisting the discovery of new information, and (2) adapting to users' reading needs.

The discovery of information within a website plays an important role for the nature of exploratory search. For instance, health websites collect many articles, and some of them are good in quality, but we have noticed that seekers may not always able to reach all of them. This implies a problem of either seekers not knowing what they want, or that they cannot effectively explore. As seen in Figure 3, seekers with the basic research tactics stay within a relatively narrow range in the information space rather than exploring other areas. In this regard, a system that understands their information needs and recommends relevant information for further reading is desirable.

Hyperlinks are also useful for relieving the problem of discovery in the health-focused exploration process. While information seekers are not clear about their concrete information needs, nor realise what content is provided in the current web site, hyperlinks are a visible mean to give hints to them on both aspects. We have identified much research on improving the search interface and information retrieval algorithms for medical websites [46-48]. We propose a clear layout of web pages with on-screen hyperlinks could help health information seekers to more easily explore relevant information. In fact, Alhenshiri et al. [49] also suggest both hyperlinks and search queries need to be considered in order to improve information gathering tasks on the web.

Our model identifies a spectrum of reading engagement, suggesting that a health website needs to adjust to both low and high reading levels. In low engagement behaviour, seekers prefer to skim and quickly read through the articles to determine the usefulness before committing to a longer reading. In this scenario, an abstract or summary could be provided for their convenience. On the contrary, people with high reading engagement may prefer a design emphasising readability, such as font size, line spacing, section navigation, etc.

As we stated above, health information seeking is, by its nature, exploratory search, hence exploration engines would be useful to engage users in the information discovery process. Software such as iFISH [3] and SeCo system [50] may be extended to provide a keyword-free exploration for consumer health information. Additional features could be added to such an engine to let the seeker choose a suitable level of readability or specifying interested health topics for fine-tuning the exploration.

\section{Conclusion}

While not every instance of health information seeking is exploratory, with this research, we have found that people take an exploratory search approach for seeking online health information in various scenarios. Exploratory seekers are found to be more engaged in the search process and potentially gain more understandings of health problems through the search. Even with these benefits, currently the support for 
exploratory health search is limited, thus we proposed to address this issue in two design directions: namely assisting the discovery of new information and adapting to reading needs.

Based on the information seeking activities in our qualitative study, we proposed a model to conceptualise health information seeking behaviours. During the information seeking process, we argue that seekers may adapt either basic or extensive research tactics, reflecting their eagerness to investigate the problem. At the same time, seekers may choose to engage in reading online information or just skim through. The combinations of research tactics and reading engagement reflect different information seeking behaviours while searching for online health information.

As with other qualitative studies, ours is subject to limitations. Participants were invited to a lab environment and performed artificial search tasks. Depending on the environment and the understanding of the tasks, the study may not truly reflect the real behaviours in their daily lives. Secondly, the time constraint of our lab study may force the participants to complete the experiment at an unusual pace. To minimize the problem, we included an interview to learn more about the daily search experience. In addition, health information seeking behaviours may vary depending on factors such as the type of diseases (i.e., acute vs. chronic) or stage of the illness. While we have presented an outcome that applies to a general group of health information seekers, it would also be valuable to extend the research to more specific groups of seekers or patients.

The findings of this paper have revealed a deeper understanding of health information needs and relevant information seeking behaviours. As a health information provider or a health website designer, more accurate and better tailor-made information can be delivered to the consumers after understanding their different information needs and information seeking behaviours. This understanding can lead to a more effective design of health websites, which provide a more pleasant experience for accessing online health information. In the future, our plan is to build a test health website with the goal of assisting the discovery of new information and enhancing reading engagement. A large scale of user study will be launched to gather feedback and evaluate the new design elements in this new website.

\section{Compliance with Ethical Standards}

Conflict of Interest: The authors declare that they have no conflict of interest.

Ethical approval: All procedures performed in studies involving human participants were in accordance with the ethical standards of the institutional and/or national research committee and with the 1964 Helsinki declaration and its later amendments or comparable ethical standards.

Informed consent: Informed consent was obtained from all individual participants included in the study.

\section{Acknowledgement}

Patrick Pang is supported by the Australian Federal and Victoria State Governments and the Australian Research Council through the ICT Centre of Excellence program, National ICT Australia (NICTA).

\section{References}

[1] Fox S, Jones S. The Social Life of Health Information; 2009.

[2] Fox S, Duggan M. Health Online 2013; 2013; http://pewinternet.org/Reports/2013/Health-online.aspx accessed on 9 Apr 2014. 
[3] Pearce J, Chang S, Kennedy G, Ely RBW, Ainley M. Search and Explore: More Than One Way to Find What You Want. 2012 Australian Computer-Human Interaction Conference (OzCHI'12); November 2630, 2012; Melbourne, Victoria, Australia; 2012. p. 469-78.

[4] Pang PC-I, Chang S, Pearce J, Verspoor K. Online Health Information Seeking Behaviour: Understanding Different Search Approaches. PACIS 2014; 24-28 Jun 2014; Chengdu, China; 2014.

[5] Marchionini G. Exploratory search: from finding to understanding. Communications of the ACM. 2006;49(4):41. doi:10.1145/1121949.1121979.

[6] Spink A, Yang Y, Jansen J, Nykanen P, Lorence DP, Ozmutlu S et al. A study of medical and health queries to web search engines. Health information and libraries journal. 2004;21:44-51.

[7] Hansen DL, Derry HA, Resnick PJ, Richardson CR. Adolescents searching for health information on the Internet: an observational study. Journal of medical Internet research. 2003;5(4):e25. doi:10.2196/jmir.5.4.e25.

[8] Andreassen HK, Bujnowska-Fedak MM, Chronaki CE, Dumitru RC, Pudule I, Santana S et al. European citizens' use of E-health services: a study of seven countries. BMC public health. 2007;7:53. doi:10.1186/1471-2458-7-53.

[9] Bessell TL, Silagy CA, Anderson JN, Hiller JE, Sansom LN. Prevalence of South Australia's online health seekers. Australian and New Zealand Journal of Public Health. 2002;26:170-3.

[10] Johnson JD, Meischke H. Women's Preferences for Cancer Information From Specific Communication Channels. The American Behavioral Scientist (1986-1994). 1991;34(6):742.

[11] McMullan M. Patients using the Internet to obtain health information: how this affects the patient-health professional relationship. Patient education and counseling. 2006;63(1-2):24-8. doi:10.1016/j.pec.2005.10.006.

[12] Nicholas D, Huntington P, Gunter B, Withey R, Russell C. The British and their use of the Web for health information and advice: a survey. Aslib Proceedings. 2003;55(5/6):261-76. doi:10.1108/00012530310498842.

[13] Yan Zhang, W-TF. Designing Consumer Health Information Systems: What Do User-Generated Questions Tell Us? FAC 2011, HCII 2011, LNAI 6780: Springer-Verlag Berlin Heidelberg; 2011. p. 536-45.

[14] Rutten LJ, Arora NK, Bakos AD, Aziz N, Rowland J. Information needs and sources of information among cancer patients: a systematic review of research (1980-2003). Patient education and counseling. 2005;57(3):250-61. doi:10.1016/j.pec.2004.06.006.

[15] Ofran Y, Paltiel O, Pelleg D, Rowe JM, Yom-Tov E. Patterns of Information-Seeking for Cancer on the Internet: An Analysis of Real World Data. PLoS ONE. 2012;7(9):e45921. doi:10.1371/journal.pone.0045921.

[16] Case DO. Looking for Information: A Survey of Research on Information Seeking, Needs, and Behaviour. Lexington: Academic Press; 2002.

[17] Wilson TD. Information behaviour: an interdisciplinary perspective. Information Processing \& Management. 1997;33(4):551-72.

[18] Wilson TD. Revisiting user studies and information needs. Journal of Documentation. 2006;62(6):680-4. doi:10.1108/00220410610714912

[19] Gorman P. Information Needs of Physicians. Journal of the American Society for Information Science. 1995;46(10):737-42.

[20] Gorman P. Information Seeking of Primary Care Physicians: Conceptual Models and Empirical Studies. Proceedings of the Second International Conference on Research in Information Needs, Seeking and Use in Different Contexts; 1999. p. 226-40.

[21] Alzougool B, Chang S, Gray K. Towards a comprehensive understanding of health information needs. electronic Journal of Health Informatics. 2008;3(2).

[22] Alzougool B, Chang S, Gray K. The nature and constitution of informal carers' information needs: what you don't know you need is as important as what you want to know. information Research. 2013;18(1). 
[23] Wilson TD. Models in information behaviour research. Journal of Documentation. 1999;55(3):249-70. doi:10.1108/EUM0000000007145

[24] Kuhlthau CC. Inside the search process: information seeking from the user's perspective. Journal of the American Society for Information Science. 1991;42(5):361-71.

[25] Ellis D. A behavioural model for information retrieval system design. Journal of Information Science. 1989;15(4-5):237-47. doi:10.1177/016555158901500406.

[26] Ellis D, Cox D, Hall K. A Comparison of the Information Seeking Patterns of Researchers in the Physical and Social Sciences. Journal of Documentation. 1993;49(4):356-69.

[27] Ellis D, Haugan M. Modelling the information seeking patterns of engineers and research scientists in an industrial environment. Journal of Documentation. 1997;53(4):384-403.

[28] Lambert SD, Loiselle CG. Health information seeking behavior. Qualitative health research. 2007;17(8):1006-19. doi:10.1177/1049732307305199.

[29] White R, Roth R. Exploratory Search: Beyond the Query-Response Paradigm. San Rafael, CA: Morgan and Claypool; 2009.

[30] Zhang Y. Exploring a Web Space for Consumer Health Information: Implications for Design. iConference 2011; Seattle, WA, USA: ACM; 2011. p. 811-2.

[31] Cartright M-A, White RW, Horvitz E. Intentions and Attention in Exploratory Health Search. The 34th Annual ACM SIGIR Conference (SIGIR'11); July 24-28, 2011; Beijing, China: ACM; 2011.

[32] Zarro M. Developing A Dual-Process Information Seeking Model for Exploratory Search. Proceedings of HCIR 2012; Cambridge, MA, USA2012.

[33] Leedy PD, Newby TJ, Ertmer PA. Practical research : planning and design / Paul D. Leedy ; with contributions by Timothy J. Newby and Peggy A. Ertmer. 6th ed. Merrill; 1997.

[34] Eysenbach G, Köhler C. How do consumers search for and appraise health information on the world wide web? Qualitative study using focus groups, usability tests, and in-depth interviews. BMJ: British Medical Journal. 2002;324(7337):573-7.

[35] Birru MS, Monaco VM, Charles L, Drew H, Njie V, Bierria T et al. Internet usage by low-literacy adults seeking health information: an observational analysis. Journal of medical Internet research. 2004;6(3):e25. doi:10.2196/jmir.6.3.e25.

[36] Buhi ER, Daley EM, Fuhrmann HJ, Smith SA. An observational study of how young people search for online sexual health information. Journal of American college health : J of ACH. 2009;58(2):101-11. doi:10.1080/07448480903221236.

[37] Creswell JW. Educational Research: Planning, Conducting, and Evaluating Quantitative and Qualitative Research. 4th Edition ed. Upper Saddle River, NJ: Merrill Prentice Hall; 2002.

[38] Braun V, Clarke V. Using thematic analysis in psychology. Qualitative Research in Psychology. 2006;3(2):77-101. doi:10.1191/1478088706qp063oa.

[39] Broder A. A taxonomy of web search. SIGIR Forum. 2002;36(2):3-10. doi:10.1145/792550.792552.

[40] Keselman A, Browne AC, Kaufman DR. Consumer health information seeking as hypothesis testing. Journal of the American Medical Informatics Association : JAMIA. 2008;15(4):484-95. doi:10.1197/jamia.M2449.

[41] Chapman K, Abraham C, Jenkins V, Fallowfield L. Lay understanding of terms used in cancer consultations. Psycho-oncology. 2003;12(6):557-66. doi:10.1002/pon.673.

[42] Choo CW, Detlor B, Turnbull D. Information Seeking on the web: an integrated model of browsing and searching. First Monday. 2000;5(2).

[43] Teevan J, Alvarado C, Ackerman MS, Karger DR. The Perfect Search Engine Is Not Enough: A Study of Orienteering Behavior in Directed Search. Proceedings of the SIGCHI Conference on Human Factors in Computing Systems 2004 (CHI'04); Vienna, Austria: ACM; 2004. p. 415-22.

[44] Li Y, Belkin NJ. A faceted approach to conceptualizing tasks in information seeking. Information Processing \& Management. 2008;44(6):1822-37. doi:10.1016/j.ipm.2008.07.005. 
[45] Jiang J, He D, Allan J. Searching, browsing, and clicking in a search session: changes in user behavior by task and over time. Proceedings of the 37th International ACM SIGIR Conference on Research \& Development in Information Retrieval; Gold Coast, Queensland, Australia. ACM; 2014. p. 607-16.

[46] Luo G, Tang C, Yang H, Wei X. MedSearch: a specialized search engine for medical information retrieval. 17th ACM conference on Information and knowledge management (CIKM '08); 2008.

[47] $\mathrm{Mu}$ X, Ryu H, Lu K. Supporting effective health and biomedical information retrieval and navigation: a novel facet view interface evaluation. Journal of biomedical informatics. 2011;44(4):576-86. doi:10.1016/j.jbi.2011.01.008.

[48] Ong E. Enabling exploratory search in UK PubMed Central: Enhancing information retrieval for the UK's biomedical and health research community. 2011 International Conference on Information Society (iSociety); 2011. p. 354-9.

[49] Alhenshiri A, Watters C, Shepherd M, Duffy J. Information Gathering Tasks on the Web : Attempting to Identify the User Search Behaviour. WEBIST 2012, LNBIP 1402013. p. 139-52.

[50] Bozzon A, Brambilla M, Ceri S, Mazza D. Exploratory search framework for Web data sources. The VLDB Journal. 2013;22(5):641-63. doi:10.1007/s00778-013-0326-x. 